plates. The chest was closed over 2 small drainage catheters placed under the pectoralis muscle.

The postoperative course was uncomplicated. On computed tomography performed on postoperative day 21 , sternal integrity was well maintained, without signs of infection (Figure 2, C). After completing 6 weeks of intravenous vancomycin therapy, she was discharged. The patient has been free of wound infections or heart failure symptoms for 6 months postoperatively.

\section{DISCUSSION}

Generally, eradication of sternal osteomyelitis requires radical debridement, sternectomy, and reconstruction of the chest wall. ${ }^{1}$ A comprehensive approach involving interposition of pedicled autologous tissue at the resected site to obliterate the dead space and fixation using multiple rigid plates to restore sternal integrity has been suggested as the ideal option for reconstructing the resected sternum in severe sternal infections. ${ }^{2,3}$

When such infections are combined with heart valvular pathologic features, several surgical approaches, either simultaneous or staged, can be considered. Staged sternal reconstruction followed by TV replacement through repeat sternotomy would have been impossible in the present case, because the titanium plates and hypervascular omental tissue would have blocked the way. Furthermore, the history of right thoracotomy will hinder an approach through this incision owing to the pleural adhesions. In contrast, valve replacement before managing the sternal osteomyelitis would have increased the risk of prosthetic endocarditis. ${ }^{4}$
Therefore, we decided on a simultaneous approach. Successful treatment of simultaneous heart valve disease and radiation-induced chest wall tumor has been reported with such a single-stage surgery. ${ }^{5}$ To the best of our knowledge, however, this is the first description of extensive sternal reconstruction combined with TV replacement for longstanding severe sternal infection. Using this aggressive approach, the chances of complications and time-consuming medical procedures might have been decreased, significantly reducing the patient's somatic and psychological stress.

\section{CONCLUSIONS}

For cases of sternal infection and concomitant cardiac valve pathologic features requiring surgery, a simultaneous approach combining valve surgery with radical resection of infected tissue and reconstruction using titanium plates and autologous tissue could be a reasonable surgical option.

\section{References}

1. Wettstein R, Erni D, Berdat P, Rothenfluh D, Banic A. Radical sternectomy and primary musculocutaneous flap reconstruction to control sternal osteitis. J Thorac Cardiovasc Surg. 2002;123:1185-90.

2. Gaetano R, Flavio F, Carmine LM, Antonello LR, Stefano M, Raffaele P, et al. Omental flap and titanium plates provide structural stability and protection of the mediastinum after extensive sternocostal resection. Ann Thorac Surg. 2010; 90:e14-6.

3. Athanassiadi K, Theakos N, Benakis G, Kakaris S, Skottis I. Omental transposition: the final solution for major sternal wound infection. Asian Cardiovasc Thorac Ann. 2007;15:200-3.

4. Sanfelippo PM, Danielson GK. Complications associated with median sternotomy. J Thorac Cardiovasc Surg. 1972;63:419-23.

5. Sachithanandan A, Dandekar U, Grimer R, Peart F, Rooney SJ. Aortic valve replacement with simultaneous chest wall reconstruction for radiation-induced sarcoma. J Card Surg. 2008;23:58-9.

\title{
Conservative management and resolution of iatrogenic type A aortic dissection in a patient with previous cardiac surgery
}

\author{
Tomasz A. Timek, MD, ${ }^{\mathrm{a}, \mathrm{b}}$ Robert Hooker, MD, ${ }^{\mathrm{a}, \mathrm{b}}$ Lawrence Patzelt, MD, ${ }^{\mathrm{a}, \mathrm{b}}$ and Gregory Bernath, MD, ${ }^{\mathrm{a}, \mathrm{c}}$ \\ Grand Rapids, Mich
}

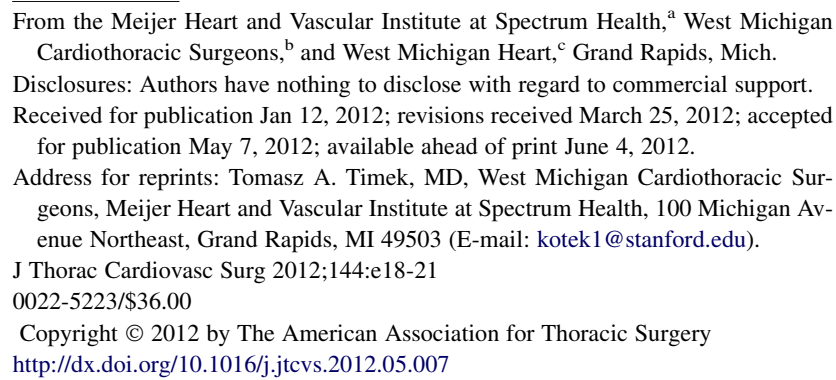

Treatment of iatrogenic acute type A aortic dissections (TAAD) remains controversial, with both surgical and percutaneous repairs advocated. We present the clinical course of a patient with previous cardiac surgery and catheterinduced acute iatrogenic TAAD that was successfully treated with conservative management.

\section{CASE REPORT}

A 55-year-old morbidly obese woman with a history of diabetes mellitus, tobacco abuse, dyslipidemia, and coronary artery disease treated twice by coronary artery bypass grafting in 2002 presented to an outside hospital 


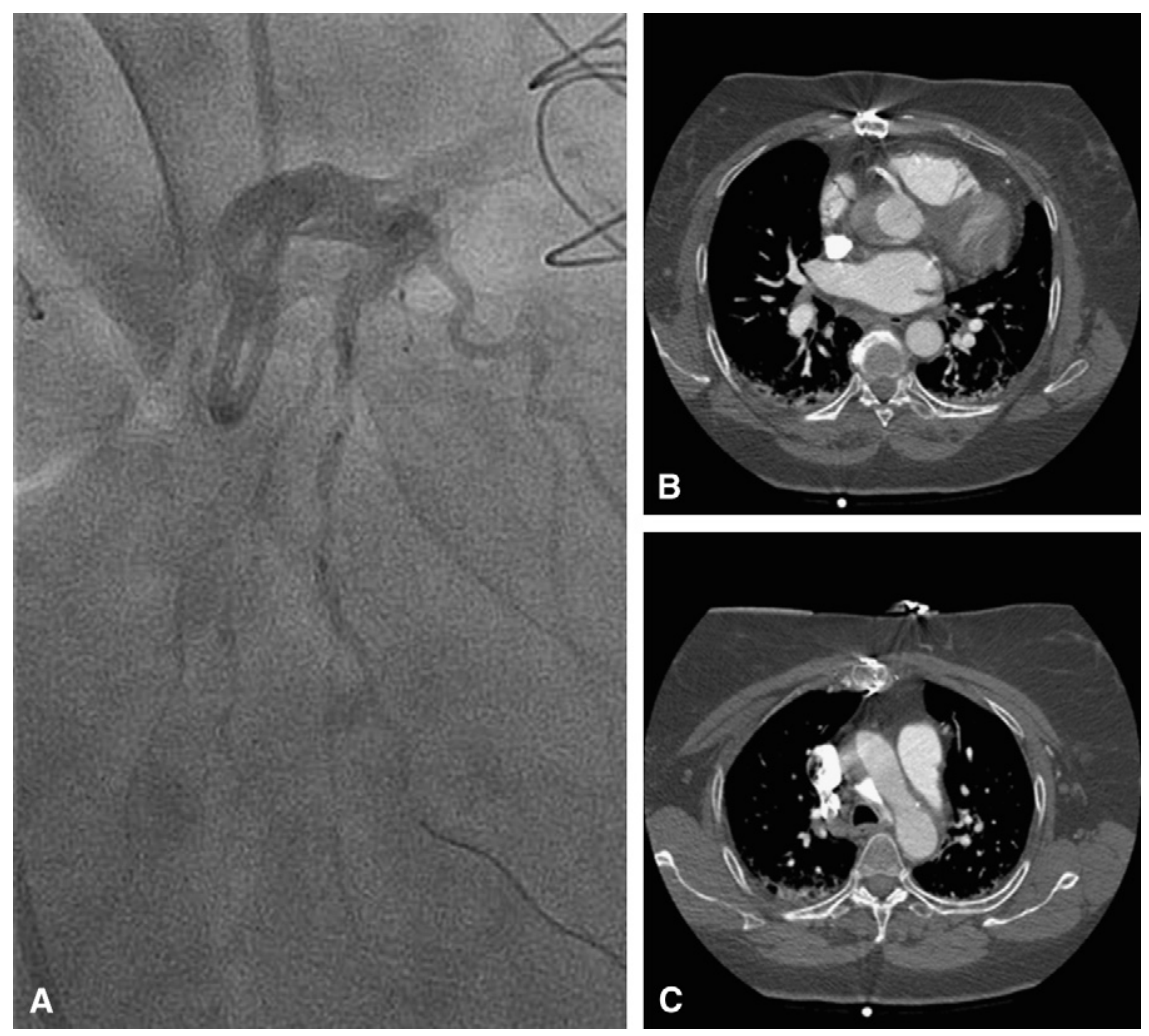

FIGURE 1. A, Coronary catheterization demonstrating type A aortic dissection after contrast injection in the aortic root. B, Computed tomography confirmed type A aortic dissection from the aortic root to the distal ascending aorta. Pooling of dense angiographic contrast was noted in the false lumen.

with acute chest pain. She reported a 6- to 7-month period of progressive unstable angina with severe substernal chest pain uncontrolled by sublingual nitroglycerin on the night of presentation. In the emergency room, the patient was diaphoretic and short of breath, with the electrocardiogram showing T-wave inversions in leads II, III, and aVF, and 2 negative serial troponin measurements. Chest radiography showed no acute cardiopulmonary process, and computed tomography of the chest with contrast did not reveal a pulmonary embolus or vascular abnormality. The patient's pain resolved in the emergency room, and coronary catheterization was performed 6 hours after admission. Coronary catheterization revealed a patent left internal mammary artery to the left anterior descending graft and a patent saphenous vein graft to the posterior descending artery. An $85 \%$ stenosis in the native second obtuse marginal artery was treated with placement of a bare metal stent. During the obtuse marginal intervention, contrast injection in the aortic root was performed, and acute TAAD was diagnosed, with suspicion of contrast injection into the aortic wall. Although the patient complained of chest pain, no evidence was found of cardiac ischemia, and the hemodynamics remained stable. The patient was immediately taken for computed tomography of the chest. The findings confirmed TAAD, with dense contrast within the false lumen (Figure 1). Because of the patient's clinical stability, morbid obesity, previous sternotomy, and mechanism of dissection, conservative management with blood pressure control, analgesics, and close observation was instituted. Echocardiography performed the following day showed normal left ventricular function with no aortic insufficiency or pericardial effusion. A computed tomography scan was repeated 2 and 6 days after the diagnosis and revealed rapid and progressive improvement in the vascular injury (Figure 2, $B$ and $C$ ). The patient was discharged on postadmission day 9 without an event. A computed tomography scan 2 months after the event showed an essentially normal ascending aorta (Figure 2,D).

\section{DISCUSSION}

Aortic root dissection induced at coronary catheterization has a reported incidence of only $0.02 \%{ }^{1}$; however, it represents a potentially lethal complication. Both surgical repair and stenting of the coronary ostia to seal the entry site have been reported as treatment options. ${ }^{1}$ However, the indications for surgical repair in otherwise hemodynamically stable patients without malperfusion or tamponade but with a history of previous cardiac surgery have not been clearly defined. Surgical repair of acute TAAD in patients with previous cardiac surgery has been 

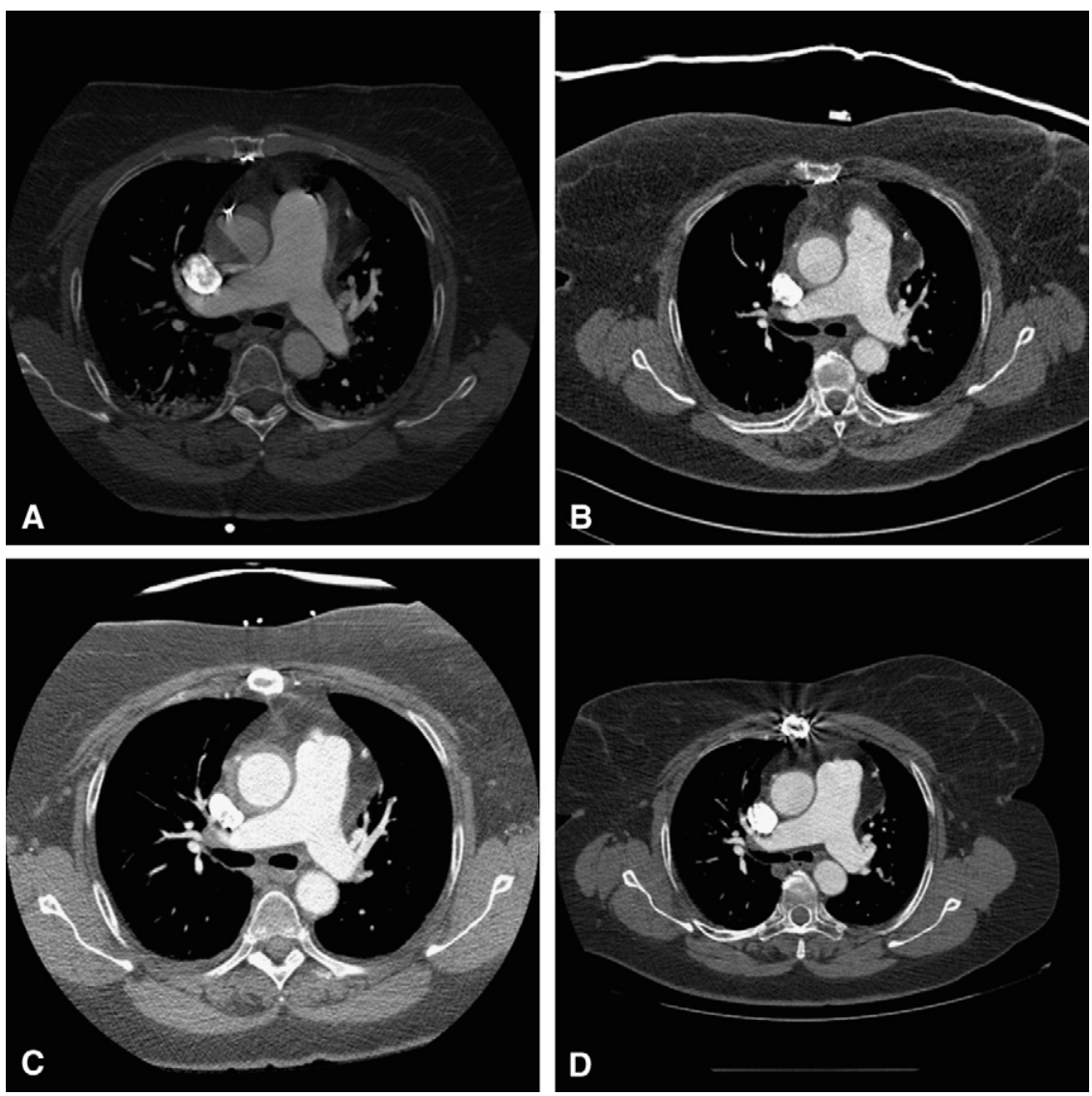

FIGURE 2. Cross-sectional computed tomography view of the ascending aorta at the level of the pulmonary artery (A) on admission, and (B) 2 , (C) 6 , and (D) 58 days after initial presentation. Near complete healing of the vascular injury was observed after 2 months.

associated with a mortality rate of greater than $50 \% .^{2}$ Even in centers of excellence, the mortality for these patients exceeds $30 \%$ and is more than twofold of that for patients with acute TAAD without previous surgery; the incidence of major cerebrovascular accidents is 4 times as high $(10 \%$ vs $2.5 \%){ }^{3}$ In contrast, in a small group of consecutive patients with TAAD and previous cardiac surgery, conservative medical management was associated with no in-hospital mortality and a $20 \%$ ( 2 of 10 patients) death rate at 14 months of mean follow-up. ${ }^{4}$ Our patient experienced an iatrogenic catheter-induced TAAD, which might portend a different prognosis than spontaneous TAAD, because the injury is often retrograde and will seal with anterograde aortic flow. A recent review of catheter-induced TAAD has confirmed this idea and revealed that expectant management for patients who did not receive a coronary stent $(39 \%$ of study population) was not associated with adverse outcomes. ${ }^{5}$ As such, the etiology of TAAD, as well as patient comorbidites, hemodynamic status, and previous surgical interventions, should be factored into the surgical decision-making process. In this context, we chose to pursue conservative management with very close observation and radiologic surveillance. Even after only 2 days, marked improvement in TAAD was demonstrated in our patient, with additional progress at 6 days and near complete healing of the aortic injury at 2 months. Similar resolution of an iatrogenic TAAD has been reported recently, although with aid of right coronary artery ostial stenting to seal the primary entry. ${ }^{6}$ This injury had nearly resolved at 30 days but was less extensive than that found in the present patient. Our report has demonstrated that resolution of this iatrogenic vascular complication is feasible without identifying or treating the primary site of injury in a selected, closely monitored patient. As such, in high-risk patients with previous cardiac surgery and iatrogenic catheter-induced TAAD who do not exhibit signs of myocardial ischemia, tamponade, or malperfusion, conservative management with close observation represents a potential treatment option.

\section{References}

1. Dunning DW, Kahn JK, Hawkins ET, O'Neill WW. Iatrogenic coronary artery dissections extending into and involving the aortic root. Catheter Cardiovasc Interv. 2000;51:387-93.

2. Stanger O, Oberwalder P, Dacar D, Knez I, Rigler B. Late dissection of the ascending aorta after previous cardiac surgery: risk, presentation and outcome. Eur J Cardiothorac Surg. 2002;21:453-8. 
3. Estrera AL, Miller CC, Kaneko T, Lee TY, Walkes JC, Kaiser LR, et al. Outcomes of acute type A aortic dissection after previous cardiac surgery. Ann Thorac Surg. 2010;89:1467-74.

4. Hassan M, Carvalho EM, Macedo FI, Gologorsky E, Salerno TA. Paradigm change in the management of patients with acute type A aortic dissection who had prior cardiac surgery. J Card Surg. 2010;25:387-9.
5. Geomez-Moreno S, Sabate M, Jimenez-Quevedo P, Vazquez P, Alfonso F, Hernandez-Antolin R, et al. Iatrogenic dissection of the ascending aorta following heart catheterization: incidence, management, and outcome. EuroIntervention. 2006; 2:197-202.

6. Garg P, Buckley O, Rybicki FJ, Resnic FS. Resolution of iatrogenic aortic dissection illustrated by computed tomography. Circ Cardiovasc Interv. 2009;2:261-3.

\section{Circumferential intimal tear in type A aortic dissection with intimo-intimal intussusception into left ventricle and left main coronary artery occlusion}

Sepehr Seyed Lajevardi, MBBS, ${ }^{\mathrm{a}}$ Karan Sian, MBBS, ${ }^{\mathrm{a}}$ Michael Ward, MBBS, PhD, ${ }^{\mathrm{b}}$ and

David Marshman, MBBS, ${ }^{\text {a }}$ Sydney, Australia

Video clip is available online.

Circumferential intimal disruption of the ascending aorta is a rare and potentially lethal occurrence. ${ }^{1}$ The intimal flap can intussuscept into the left ventricular outflow tract, causing significant aortic regurgitation, and may impair coronary perfusion of the coronary arteries (Figure 1). We discuss a case of type A aortic dissection with circumferential intimal tear and intimo-intimal intussusception causing intermittent total left main coronary artery (LMCA) occlusion.

\section{CLINICAL SUMMARY}

A 73-year-old woman who was a nursing home resident was found lying on the floor amnesic to events. She was taken to the emergency department with a symptom of central chest pain. Her medical history was significant for longstanding untreated hypertension, heavy smoking, and mild Alzheimer's dementia. On examination, the patient had a heart rate of 50 beats/min and a systolic blood pressure of $70 \mathrm{~mm} \mathrm{Hg}$. Investigations showed intermittent, widespread ST depression and T-wave inversion on

\footnotetext{
From the Departments of Cardiothoracic Surgery ${ }^{\mathrm{a}}$ and Cardiology, ${ }^{\mathrm{b}}$ Royal North Shore Hospital, University of Sydney, Sydney, Australia.

Disclosures: Authors have nothing to disclose with regard to commercial support.

Received for publication March 29, 2012; accepted for publication May 7, 2012; available ahead of print June 7, 2012.

Address for reprints: Sepehr Seyed Lajevardi, MBBS, Department of Cardiothoracic Surgery, Royal North Shore Hospital, Pacific Highway, St Leonards NSW 2065, Sydney, Australia (E-mail: sepehrlaj@gmail.com).

J Thorac Cardiovasc Surg 2012;144:e21-3 0022-5223/\$36.00

Crown Copyright $\odot 2012$ Published by Elsevier Inc. on behalf of The American Association for Thoracic Surgery

http://dx.doi.org/10.1016/j.jtcvs.2012.05.010
}

electrocardiogram, and an increased troponin $\mathrm{T}$ level of $93 \mathrm{ng} / \mathrm{L}$ (normal, $<14 \mathrm{ng} / \mathrm{L}$ ) on blood test results.

With an initial diagnosis of acute coronary syndrome, urgent coronary angiography was performed (Figure 2, A, and Video $1, A$ and $B$ ). There appeared to be complete proximal occlusion of the LMCA. However, the appearance of "late ghosting" of the LMCA and preserved left ventricular function was not consistent with a total LMCA occlusion. On aortography, there was evidence of extensive Stanford type A aortic dissection, and left ventriculography revealed diastolic prolapse of the aortic intima through the left ventricular outflow tract causing severe aortic regurgitation. The appearance of complete occlusion of the LMCA was thought to be due to intermittent occlusion of the left coronary ostium by the intussuscepting dissection flap or placement of the angiographic catheter in the inner intima (intussusceptum).

The patient was taken urgently to the operating room, and the intraoperative transesophageal echocardiography (TEE) showed circumferential dissection of the ascending aorta, with the cylinder-shaped intimal flap intussuscepting back into the left ventricle causing severe aortic regurgitation (Figure 2, B, and Video 2, $A$ and $B)$. TEE also showed mild left ventricular hypertrophy and mild inferior wall hypokinesis. Aortotomy revealed a circumferential intimal tear originating approximately $3 \mathrm{~cm}$ from the sinotubular junction with the false lumen extending to the aortic root. Valve leaflets and coronary ostia had a normal appearance. The patient underwent successful aortic valve resuspension with replacement of the affected ascending aorta. Postoperative computed tomography aortography revealed that the dissection extended down to the iliac vessels involving multiple aortic branches (Figure 3), which was managed conservatively. She progressed well postoperatively and was discharged to respite care. 